Heylen, E., Labeeuw, W., Deconinck, G., Van Hertem, D. (2016). "Framework for evaluating and comparing performance of power system reliability criteria".

This is the author's version of an article that has been accepted for publication in IEEE Transactions on Power Systems. Changes are made to this version by the publisher prior to publication.

Digital Object Identifier: 10.1109/TPWRS.2016.2533158

URL: http://ieeexplore. ieee.org/xpls/abs_all.jsp?arnumber=7428968

(C)2016 IEEE. Personal use of this material is permitted. Permission from IEEE must be obtained for all other users, including reprinting/ republishing this material for advertising or promotional purposes, creating new collective works for resale or redistribution to servers or lists, or reuse of any copyrighted components of this work in other works. 


\title{
Framework for Evaluating and Comparing Performance of Power System Reliability Criteria
}

\author{
Evelyn Heylen, Graduate student member, IEEE, Wouter Labeeuw, Geert Deconinck, Senior member, IEEE, \\ and Dirk Van Hertem, Senior member, IEEE
}

\begin{abstract}
Evolutions in the power system challenge the manner in which power system reliability is managed. In particular, currently used reliability criteria, typically the deterministic N-1 criterion, are increasingly inadequate. Moving to an alternative approach is difficult as quantifying benefits is hard in a multifaceted environment and system operators are reluctant to move away from the easy and transparent existing criterion. This paper presents a generic framework to evaluate and compare socioeconomic and reliability performance of power system reliability criteria, focussing on the short term decision making process of transmission system operators (TSO). The framework can also be used to tune the parameters of reliability criteria. Short term operational planning and real time operation TSO decision making processes are simulated considering various reliability criteria. The framework is applied to a 5 node test system and the 24 node IEEE reliability test system, showing that the applied probabilistic reliability criterion outperforms deterministic $\mathbf{N}$ 0 and N-1 approaches in those systems in terms of expected reliability and socio-economic indicator values. The effect is larger in the bigger system with more operational flexibility.
\end{abstract}

Index Terms-Power system operation, power system reliability, reliability criterion, reliability management.

\section{NOMENCLATURE}

$i \quad$ Set of generators in the system

$j \quad$ Set of loads in the system

$k \quad$ Set of assessed real time system states in evaluation

$q \quad$ Set of credible real time states used in operational planning

$s \quad$ Set of load forecast values

$C_{L C, q} \quad$ Cost of load curtailment per credible state $q$

$C_{\text {redisp,q }}$ Redispatch cost per credible state $q$

$C_{\text {res }} \quad$ Cost of reserve contracting

$c_{G, i} \quad$ Marginal cost of generation

$c_{G, \text { red,i }}^{+} \quad$ Price of upward redispatch of generator $i$

$c_{G, \text { red }, i}^{-} \quad$ Price of downward redispatch of generator $i$

$c_{r e s, i} \quad$ Price of reserve provision of generator $i$

$P_{G, i, q} \quad$ Active power production of generator $i$ in credible state $q$

$P_{G, \text { sched }, i}$ Scheduled generation of generator $i$

$P_{G, \max , i}$ Generation capacity of generator $i$

$P_{\text {shed }, j, q}$ Expected load curtailment of load $j$ in credible state $q$

$P_{\text {shed,j,k|s }}$ Real time load curtailment of load $j$ in state $k$ given load forecast $s$

$\Delta P_{G, i, q} \quad$ Expected total redispatch of generator $i$ in credible state $q$

$\Delta P_{G, i, q}^{+} \quad$ Expected upward redispatch of generator $i$ in credible state $q$

The work of Evelyn Heylen is supported by the Research Foundation Flanders (FWO)

The research leading to these results has received funding from the European Union Seventh Framework Programme under Grant Agreement No 608540 .

The authors are with the Department of Electrical Engineering, Div. ELECTA, KU Leuven, Leuven, Belgium. The Electa research group is a founding member of EnergyVille. Contact: evelyn.heylen@esat.kuleuven.be, dirk.vanhertem@esat.kuleuven.be

\author{
$\Delta P_{G, i, q}^{-} \quad$ Expected downward redispatch of generator $i$ in \\ credible state $q$ \\ $\mathrm{Pr}_{q} \quad$ Probability of occurrence of state $q$ \\ $\operatorname{Pr}_{k \mid s} \quad$ Conditional probability of real time state $k$ given \\ load forecast $s$ \\ $\mathrm{Pr}_{s} \quad$ Probability of load forecast $s$ \\ $R_{r e q} \quad$ Reserve requirement \\ $r_{i} \quad$ Amount of reserves contracted of generator $i$ \\ $R R_{i} \quad$ Ramp rate limit of generator $i$ \\ $V o L L_{j} \quad$ Value of lost load of load $j$
}

\section{INTRODUCTION}

$\mathrm{T}$ HE POWER system has evolved considerably over the last decades. These evolutions include the increased use of intermittent generation, arising opportunities for demand side management and energy storage, liberalization of the energy market and unbundling, ... resulting in more uncertainty in the system. Furthermore, lack of investments in generation and transmission capacity due to uncertainty and slow permission procedures puts more stress on the system. Therefore, the question arises whether currently used deterministic reliability criteria, which were developed with a traditional system in mind, can handle challenges posed by evolutions in the system in a cost effective way.

The N-1 criterion $^{1}$, or a specific derivation thereof, is commonly used in power systems. The $\mathrm{N}-1$ criterion has proven its success over the last decades by leading to acceptable results in terms of reliability, while being transparent and easy to use [1]. However, its shortcomings, such as the fact that only single contingencies are taken into account, while considering them all equally probable and equally severe [2]-[6], might lead to stressed situations in the future. Furthermore, an $\mathrm{N}$ 1 approach does not give an economic incentive to strive for a balance between cost of reliability and reliability value (For example, do remote regions with only a few houses and low interruption costs require the same level of reliability as a densely populated city, even at a high cost?). Moreover, it is not obvious to the operator how reliability and cost are balanced in various operating conditions. Probabilistic reliability management can overcome shortcomings of an $\mathrm{N}-1$ approach at the cost of higher complexity and computational requirements [4], [7]. However, a trade-off between costeffectiveness, practicality of reliability management and social acceptance needs to be made.

In order to convince stakeholders in the power system to use alternative reliability criteria, it is important to weigh

${ }^{1}$ Several definitions are available. Definition according to ENTSO-E: The rule according to which elements remaining in operation within TSO's responsibility area after a contingency from the contingency list must be capable of accommodating the new operational situation without violating operational security limits. 
benefits of using alternatives over various operating states in an objective way, over extended time, considering locational effects. It is important to compare not only final system states, but also the reliability decision related trajectories according to the reliability criteria. Existing studies only focus on limited parts of the problem without integrating them. They propose alternative decision making tools [8], [9], compare probabilistic and deterministic security assessments [4], [7] or evaluate and compare performance of various reliability management approaches focussing on the interdependence between market performance and system security [10]. SAMREL is an integrated approach for reliability of electricity supply analysis [11]. However, it focusses on long term planning aspects and considers only deterministic reliability criteria and a limited amount of candidate decisions in the short term.

This paper presents a generic framework for evaluating and comparing both socio-economic and reliability performance of power system reliability criteria and their management. The main contribution of this work is the modular design of a framework that allows to quantify the performance of various reliability criteria by evaluating both the final system state as well as the reliability management process. This requires a generic way of combining various aspects of power system operation, including market clearing, determination of operational planning (OP) decisions and taking real time (RT) actions. The framework is easy to expand and the amount of detail can be increased or reduced in a transparent way. The implementation is made in MATLAB. Due to its modular and generic design, modules of the platform can be substituted by existing tools or more advanced implementations with similar functionality.

A large scale implementation of the framework based on the presented theoretical design can be used to guide the regulator and transmission system operators towards cost-effective reliability criteria. The framework can help them making a trade-off between optimality of social surplus, practicality of reliability management and social acceptance. Possible changes of using alternative reliability criteria can be quantified on stakeholder level and on system level. Another important feature of the framework is the tuning of parameters of reliability criteria.

The main focus of this paper is on the theoretical background of the framework for evaluating and comparing performance of power system reliability criteria, rather than on conclusions in terms of performance of various reliability criteria. An overview of the framework with its possibilities and its objectives as well as possible applications are described in section II. Section III focuses on the simulation of the short term decision making process and explains the various modules, while section IV and $\mathrm{V}$ respectively focus on the evaluation and comparison of the performance of reliability criteria. In section VI, the framework is applied to a 5 node test system and the IEEE 24 node reliability test system. Three different reliability criteria are compared. In this illustrative case study, a basic configuration of the framework is used. Finally, section VII concludes the paper.

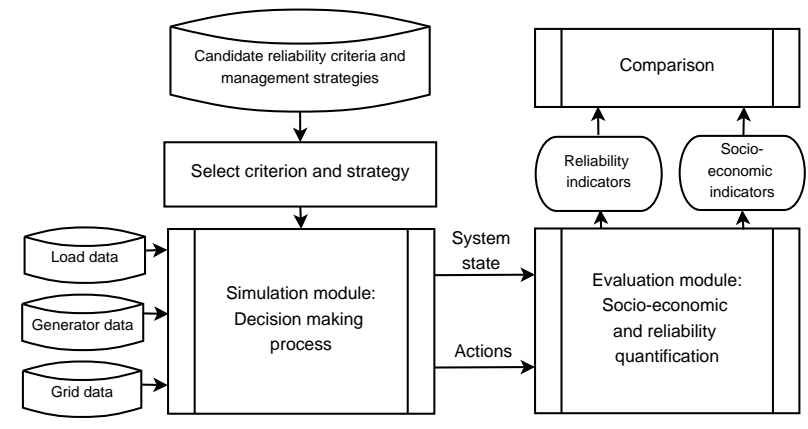

Fig. 1. Overview of the framework for evaluating and comparing performance of power system reliability criteria and their management [13].

\section{OVERVIEW AND OBJECTIVES OF THE FRAMEWORK}

Evaluating and comparing performance of power system reliability criteria requires three main tasks:

1) Simulation of the decision making process considering a particular reliability criterion, including evaluation of possible candidate decisions,

2) Quantifying performance of various reliability criteria in terms of reliability and socio-economic indicators, both at system level and stakeholder level,

3) Comparing performance of various reliability criteria. Interlinking between these three tasks in the framework is shown in Fig. 1. Firstly, a reliability criterion is selected from a list of candidate reliability criteria. The criterion is satisfied by taking appropriate reliability decisions. Resulting actions lead to a final operational state of the power system, which is evaluated together with the actions taken. To allow benchmarking, this process is repeated with identical load, generator and grid data, but for a known reliability criterion. The postprocessing stage compares results for various assumptions and reliability criteria. Results of the comparison can be used in order to conclude whether alternative reliability criteria lead to a higher level of social surplus ${ }^{2}$ in the electricity system and to tune parameters of reliability criteria, such as risk level for instance.

\section{Simulation OF TSO DECISION MAKING PROCESSES}

The framework presented focuses on the short term decision making processes of the transmission system operator, i.e. day ahead up to real time. The decision making process consists of two stages:

- Operational planning stage or scheduling

- (Near) real time operation stage

Scheduled generation commitments at the different nodes in the system are obtained from the day ahead market clearing. In case of a copper plate day ahead market, security constraints and power system limits are not taken into account in the market clearing. This might require planning of actions such as redispatch, phase shifting transformer tap changing, etc. in

${ }^{2}$ Social surplus is the generally accepted measure in economics to compare the impact of policy measures, in our case power system reliability criteria [12]. 


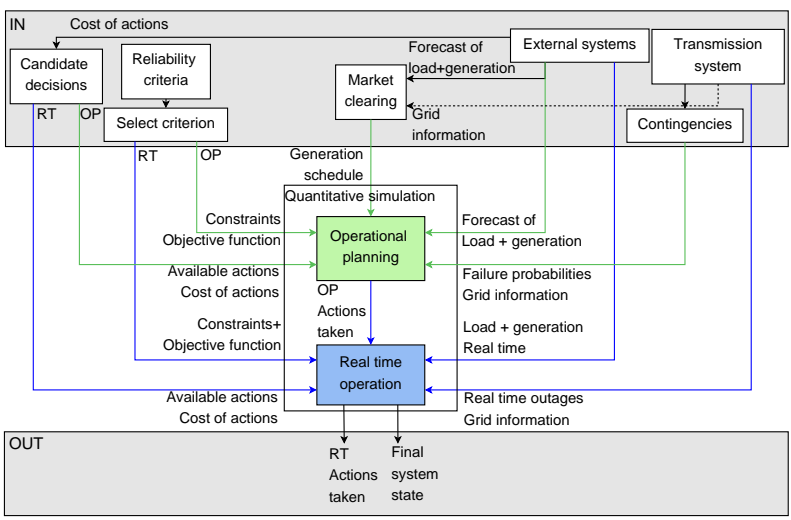

Fig. 2. Overview of the simulation module together with its outputs and modules serving the inputs. 'RT' and 'OP' refer to data of a particular module regarding real time operation and operational planning respectively.

order to satisfy the reliability criterion and the system limits. Scheduling of these actions is done in the operational planning stage, considering the unit commitment schedule resulting from the market clearing process based on forecasted load and generation and the reliability criterion. Measures taken during the operational planning stage are called preventive actions, which are taken before real time in order to achieve security and improve the ability to withstand the possible effects of potential contingencies [14].

The operational planning stage is based on expected system states that might differ from the real time system state. Therefore, a final decision stage based on the real time realizations of demand, generation capacity and outages needs to be included that allows for curative actions to keep the system within limits. Outcomes of this real time decision stage are called curative actions, needed in real time to satisfy the applied reliability criterion and meet operational limits. Links between the two decision stages and the various modules in the implementation are shown in Fig. 2. The 'IN'-block combines modules delivering input for the simulation module as given in Fig. 1. The 'OUT'-block contains outputs of the simulation module. Following subsections describe the modules.

\section{A. External systems}

The 'external systems'-module contains systems over which the TSO has limited control:

- Generation, of which the module contains forecast and real time generation capacity, failure and repair rates, marginal costs of generation, capacity available for reserves, cost of reserve provision and redispatch cost;

- Load, of which the module contains forecast and real time demand in the system, value of lost load (VoLL);

- Market, of which the module contains the type of the market, i.e. constrained market or copper plate market;

- Weather, of which the module contains forecast and real time values of wind speed, wind direction, solar power, temperature at different points in the system, etc.

Spatio-temporal correlation in meteorological data and load data can be included using correlation matrices. Impact factors of weather parameters on operational limits, e.g. dynamic line rating, and reliability data, e.g. failure rates, can also be provided by this module.

All these external systems are interdependent and will serve as an input for the TSO decision making processes of operational planning and real time operation.

\section{B. Transmission system}

The 'transmission system'-module contains parameters related to the grid and its components:

- Topology of the grid, i.e. connections between the nodes, location of load and generation, location of flexible devices, switchgear, etc.

- Characteristics of system components, i.e. operational limits of lines and flexible devices, settings of flexible devices, impedance of lines, status of components, etc.

- Reliability data of system components, i.e. failure and repair rates of lines and flexible devices, etc.

Reliability data and characteristics of system components are in practice influenced by weather conditions as well as decisions taken in earlier time horizons, such as maintenance actions influencing the failure rate of components.

Grid information coming from the 'transmission system'module is used to build the simulation matrix of the base case system. Additional cases of credible system states that might need to be considered simultaneously in the quantitative simulation of the decision making process according to a particular reliability criterion are added to this matrix. Furthermore, the 'transmission system'-module provides operational limits of system components that are considered as constraints in the quantitative simulation.

\section{Contingencies}

The 'contingencies'-module gets failure and repair rates of system components as an input from the 'transmission system'-module and serves three purposes:

1) Determination of the probability of occurrence of various states of a particular system component. Due to non-exponentially distributed repair times, systems are in general non-Markovian. Moreover, bad weather conditions or bad maintenance might result in non-constant failure rates. This requires methods of supplementary variables, device of stages, semi-Markov processes [15] or simulation techniques to be applied. However, if assumptions of exponentially distributed time to failure and repair times do not imply significant differences, approximate methods, such as a Markov process, may be used [16].

2) Determination of the probability of an outage of a particular (combination of) system component(s)

3) Contingency selection

The probability of occurrence of an outage of a particular (combination of) system component(s) can be directly used in the objective function specified by the reliability management strategy in order to weigh outcomes for various expected system states in the decision making process. Furthermore, 
TABLE I

OVERVIEW OF POSSIBLE CANDIDATE DECISIONS IN OPERATIONAL PLANNING AND REAL TIME OPERATION

\begin{tabular}{ll}
\hline Real time operation & Operational planning \\
\hline No action & No action \\
Generation redispatch & Contracting reserves \\
Load curtailment & Contracting flexibility \\
Bus bar and line switching & Generation rescheduling \\
Transformer tap changing & + Actions real time operation \\
\hline
\end{tabular}

probabilities are needed in analytical evaluation techniques in order to weigh the performance of a particular outcome of the decision making process in the overall performance of the reliability criterion and its management. In order to reduce the computational burden of the analytical evaluation techniques, appropriate contingency selection methods can be applied [15], [17], [18].

The probabilities can also be used to sample the status of various transmission system components according to their availability resulting in time series of the status of all components. These samples are useful in Monte Carlo simulations, which can be applied to evaluate the performance of a particular reliability criterion.

\section{Candidate decisions}

The time horizon determines the candidate reliability decisions of a transmission system operator. In the short-term horizon from D-1 up to real time, candidate decisions differ between the operational planning stage and the real time operation stage. An overview of possible candidate decisions is given in table I. Candidate decisions available in real time are also considered in the decision making process of operational planning as they serve as additional flexibility that is still available in real time.

The 'candidate decisions'-module provides constraints and cost functions in parametric form for the simulation of the two decision stages. Values for the parameters in these functions come from the 'external systems'- and 'transmission system'modules.

\section{E. Reliability criteria}

Outputs of the 'reliability criteria'-module are constraints that need to be satisfied and the objective function according to which the reliability needs to be managed, both in parametric form. Constraints posed by the reliability criterion consist of limits on reliability indicators, such as expected load curtailment, as well as limits on physical quantities in the system, e.g. branch flow limits that cannot be violated in particular credible system states. Credible system states are selected based on two attributes: plausibility and likelihood [19]. Depending on the characteristics of the reliability criterion, they can be defined deterministically, e.g. all contingency cases up to N-k system states, or probabilistically, e.g. all likely system states up to a cumulative probability of occurrence of $\mathrm{X} \%$ [13]. Depending on the criterion and the decision stage, credible system states can consider possible outages of transmission system components as well as real time realizations of demand and generation capacity, which are uncertain intra-day. Power flow constraints for all credible system states are included in the optimal power flow (OPF) formulation and those are coupled by coupling constraints based on ramp rate limits among others. Constraints can also be of a stochastic nature, e.g. chance constraints [20], which need to be satisfied in a particular percentage of the cases, or can focus on the $\alpha$ percentile of worst outcomes in order to limit consequences of bad outcomes [21].

Social surplus is an appropriate metric to rank performance of various available reliability decisions. It consists of consumer surplus, i.e. the difference between willingness to pay (WTP) and the price paid for the good, and producer surplus, i.e. all profits in the market. In case of the electricity system, both generators and grid operators are considered as producers [12]. However, not all data to determine social surplus are known by the TSO when reliability decisions must be taken. Therefore, it is not possible in practice to operate the system using the ideal objective function that maximizes social surplus.

In order to mimic TSOs' decision making behavior it is important to take into account TSOs' data availability. Artificial rules based on physical or reliability indicators or alternative socio-economic indicators need to be developed and applied in practice ${ }^{3}$. Alternative objective functions aim at minimizing total system cost, possibly taking into account weights for the probability of occurrence of credible system states [8], [22]. Conditional value at risk $(\mathrm{CVaR})$ or value at risk (VaR) can also be used as objective function in order to include risk aversion of the decision maker [12], [20].

\section{F. Quantitative simulation}

The 'quantitative simulation'-module performs the decision making process at the two decision stages. For the operational planning decision stage, the decision making process is implemented as a two stage stochastic security constrained optimal power flow taking into account:

- Constraints posed by the reliability criterion regarding credible real time system states

- Operational limits in the transmission system

- Available candidate decisions

- Power flow constraints

If multiple (combinations of) decisions satisfy reliability and operational limits, possible (combinations of) decisions are ranked based on the objective function as specified by reliability management. The outcome of the optimization will be operator actions that are optimally taken ahead of real time in order to satisfy the reliability criterion and operational limits.

In the real time decision making process, similar types of constraints are taken into account, but the uncertainty is reduced. The outcome of the operational planning decision stage is used as an input for the real time operation stage. A security constrained optimal power flow (SCOPF) calculation

\footnotetext{
${ }^{3}$ GARPUR, http://www.garpur-project.eu
} 
is used, which results in curative actions that need to be taken in real time in order to obtain an acceptable reliability level in the system as defined by the reliability criterion.

Failure of curative actions in real time operation can be considered in the decision making process by including an additional decision stage, introducing additional states [22]. Furthermore, additional stages in the optimization process can be used to include pseudo-dynamic behavior of the system in order to guarantee that constraints are satisfied in the postcontingency state before and after curative actions are fulfilled [23].

The quantitative simulation can use either an AC SCOPF or a DC SCOPF with reduced computational burden. In order to check satisfaction of reactive power limits, branch flow limits and voltage limits in the latter case, the outcome of the DC SCOPF can be used as an input for an AC power flow check. If constraints are not satisfied, actions can be taken based on heuristics [11]. Alternatively, an iterative approach that complements or substitutes the optimization can be used as well. The outcome of the quantitative simulation is the final grid state and the actions taken by the TSO aiming at a secure and operational system.

\section{Performance eValuation}

Performance of reliability criteria is evaluated using an expost assessment [12]. The ex-post assessment evaluates the decisions taken according to a particular reliability criterion after they are put in practice. Next to evaluating the final state, it is important to evaluate the trajectory leading to this final state. The final system state and actions taken can be quantified in terms of physical indicators, such as energy not served (ENS), interruption duration, reserves provided and used, remaining capacity of generators and lines, etc. They also lead to costs and benefits for different stakeholders in the system, which can be quantified in terms of socio-economic quantities, such as social surplus, total cost, interruption cost, etc. Indicators vary with changing operating states and can be expressed in instantaneous, average, extreme or median values.

In order to make a decent evaluation of performance, it is important to consider sufficient system states in the analysis of a reliability criterion, taking into account their probability of occurrence. Several assessment methods exist. An analytical approach assesses in theory all possible system states. Even the states outside the credible system states defined by the criterion should be assessed. However, this might be cumbersome in large transmission systems. An approximate analytical state enumeration method might be an alternative, in which only a predefined number of system states is assessed [15], [17], [18].

Alternatively, a Monte Carlo approach can be applied. This approach uses combinations of samples of the status of all system components according to their availability. However, a sufficiently high number of samples is needed, which requires an appropriate convergence or stopping criterion. Hybrid approaches combine aspects of simulation techniques and enumeration methods [24].

\section{COMPARING PERFORMANCE OF RELIABILITY CRITERIA}

Different reliability criteria will lead to different decisions implying a different performance. If the same process with equal input data is repeated for various reliability criteria, performances can be compared. Comparing the performance of reliability criteria is preferably done on a relative scale. Therefore, benchmarking against an existing reliability criterion is a useful approach. Quantification of performance of reliability criteria in terms of reliability indicators and socio-economic indicators as done in this framework allows for a numerical measure of change in performance obtained by using an alternative reliability criterion in specific circumstances. However, it might be that no unique optimal reliability criterion exist as the performance of reliability criteria strongly depends on various system parameters, such as value of lost load, system robustness, etc. [13]. Therefore, reliability criteria with changing parameters as a function of system characteristics and conditions might be more cost effective.

\section{APPLICATION OF THE FRAMEWORK}

A case study for a 5 node test system [13], [25] illustrates how the framework can be used. An application of the framework to the IEEE 24 node reliability test system [26] demonstrates its scalability. The case study uses a particular configuration of the framework, i.e. the applied simulation method (i.e. AC vs DC-OPF), reliability actions that are considered, etc. However, this configuration can be adapted to the needs of the user by enabling more advanced methods and reliability actions.

\section{A. Test systems}

Generator data used in the 5 node system are summarized in table II. Forecast of total system load follows the load profile as given in Fig. 3(a) [25].

TABLE II

OVERVIEW OF DATA OF THE 5 NODE TEST SYSTEM

\begin{tabular}{lcccc}
\hline Gen-No. & Node & $P_{G, \max , i}[\mathrm{MW}]$ & $c_{G, i}[€ / \mathrm{MWh}]$ & $c_{r e s, i}[€ / \mathrm{MW}]$ \\
\hline 1 & 1 & 40 & 79 & 44.5 \\
2 & 1 & 40 & 77 & 43.5 \\
3 & 1 & 10 & 0.02 & 5 \\
4 & 1 & 20 & 0.04 & 5 \\
5 & 2 & 40 & 90 & 50 \\
6 & 2 & 20 & 76 & 43 \\
7 & 2 & 20 & 0.01 & 5 \\
8 & 2 & 20 & 0.03 & 5 \\
9 & 2 & 20 & 0.05 & 5 \\
10 & 2 & 5 & 99 & 54.5 \\
11 & 2 & 5 & 78 & 44 \\
\hline
\end{tabular}

Data of the IEEE 24 node reliability test system can be found in literature $[26]^{4}$. Generator costs are considered to be linear for all generators in both systems. If no value of lost load is mentioned, a value of $€ 17500 / \mathrm{MWh}$ is used [27]. 20\% of load is considered to be available for demand side response at a cost. The remaining part of the load can only be curtailed in emergency cases. Branches and generators are represented as

\footnotetext{
${ }^{4}$ Node 7 and 8 are aggregated at node 8 to obtain a $\mathrm{N}-1$ compliant lay-out.
} 

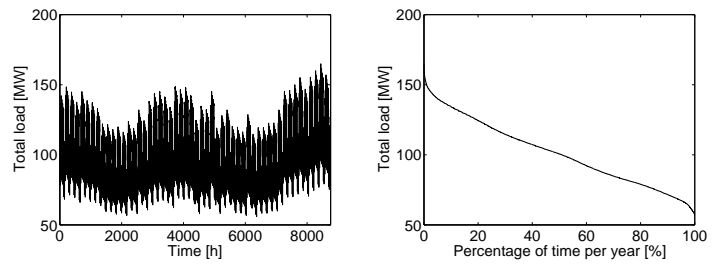

(a) Sequential total load profile for (b) Load duration curve of the total the 5 node test system load profile
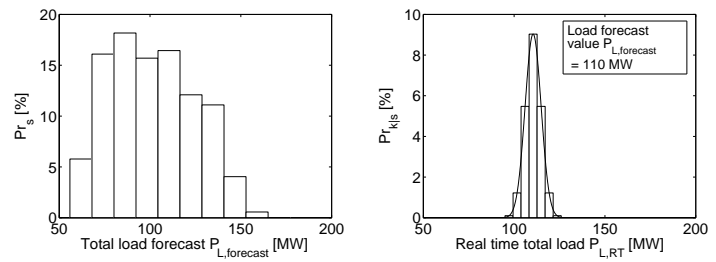

(c) Discrete probability density (d) Discrete probability density function of expected total system function of real time total system load forecast load for given load forecast

Fig. 3. Overview of total load representations used in the evaluation

two state component models. Branches are assumed to have constant failure rates and repair times, while generators are assumed to have constant failure and repair rates [25], [26]. The impact of non-exponentially distributed repair times on the state probabilities is verified using a Monte-Carlo simulation technique and is determined to be not significant for this illustrative case study [16].

Results shown hold for the 5 node system unless stated otherwise.

\section{B. Overview of the case study}

Three different reliability criteria [13] are applied to the single area 5 node and 24 node test systems:

- N-0 criterion, i.e. no load curtailment or violation of operational limits allowed in the $\mathrm{N}-0$ system state for the forecast value of real time load,

- N-1 criterion, i.e. no load curtailment or violation of operational limits allowed in all contingency cases up to $\mathrm{N}-1$ for the forecast value of real time load,

- Probabilistic reliability criterion, i.e. minimal expected system cost taking into account contingency cases up to a cumulative probability of $99 \%$ and 7 load scenarios with their respective probability of occurrence.

A simplified TSO decision making process aiming at minimal system cost while satisfying constraints posed by the reliability criterion is implemented. MATPOWER's [28] extensible optimal power flow formulation is used for simulating the decision making process. The quantitative simulation is based on a DC SCOPF. Outcomes of the DC-SCOPF are used as input for an AC power flow to check the results. The simulation is non-sequential. Start-up times and shut-down times of generators are ignored. A constrained market clearing is considered.
The objective function for the operational planning stage is:

$$
\begin{gathered}
\min \sum_{i=1}^{G}\left[P_{G, \text { sched }, i} \cdot c_{G, i}\right]+\sum_{i=1}^{G}\left[c_{r e s, i} \cdot r_{i}\right]+\ldots \\
\sum_{q}\left[\operatorname{Pr}_{q} \cdot\left(C_{\text {redisp }, q}+C_{L C, q}\right)\right]
\end{gathered}
$$

Generation redispatch and load curtailment costs can be quantified as follows in the objective function:

$$
\begin{aligned}
& C_{\text {redisp }, q}=\sum_{i=1}^{G}\left[c_{G, r e d, i}^{+} \cdot \Delta P_{G, i, q}^{+}+c_{G, r e d, i}^{-} \cdot \Delta P_{G, i, q}^{-}\right] \\
& C_{L C, q}=\sum_{j=1}^{L} V_{o L L} \cdot P_{\text {shed }, j, q}
\end{aligned}
$$

Reserve contracting is considered in the operational planning stage, which requires following constraints on top of Kirchoff's first law, branch flow limits and voltage angle limits [29]:

$$
\begin{array}{ll}
\sum_{i=1}^{G} r_{i} \geq R_{r e q} & \forall i \\
r_{i}+P_{G, \text { sched }, i} \leq P_{G, \text { max }, i} & \forall i \\
0 \leq r_{i} \leq R R_{i} & \forall i \\
r_{i}-\Delta P_{G, i, q}^{+} \geq 0 & \forall i, q
\end{array}
$$

Similar constraints can be considered for downward reserve contracting, focussing on downward redispatch requirements and minimal generation of each generator. The reserve requirement is the same for the three reliability criteria and asks for an upward reserve capacity equal to the largest generator in the system.

Load curtailment and generation redispatch are also considered in the operational planning stage as available actions in real time. However, limits are put on the amount of redispatch between the scheduling stage and the expected real time system state $q$ based on the ramp rate limits of the generators:

$$
\begin{array}{ll}
-R R_{i} \leq\left|\Delta P_{G, i, q}\right| \leq R R_{i} & \forall i, q \\
\Delta P_{G, i, q}=P_{G, i, q}-P_{G, \text { sched }, i} & \forall i, q
\end{array}
$$

In order to linearize the problem, following equations are used in which $\Delta P_{G, i, q}^{+}$and $\Delta P_{G, i, k}^{-}$are considered as an upper bound on positive and negative redispatch, respectively:

$$
\begin{array}{ll}
\Delta P_{G, i, q}=\Delta P_{G, i, q}^{+}-\Delta P_{G, i, q}^{-} & \forall i, q \\
P_{G, i, q}-P_{G, s c h e d, i} \leq \Delta P_{G, i, q}^{+} & \forall i, q \\
P_{G, s c h e d, i}-P_{G, i, q} \leq \Delta P_{G, i, q}^{-} & \forall i, q \\
0 \leq \Delta P_{G, i, q}^{+} \leq R R_{i} & \forall i, q \\
0 \leq \Delta P_{G, i, q}^{-} \leq R R_{i} & \forall i, q
\end{array}
$$

Load curtailment is included in the OPF formulation as negative generation in the system at the cost of value of lost load, which is assumed to be sufficiently high if no load curtailment is allowed in particular credible system states.

In the real time operation stage, cost of scheduled generation $\sum_{i=1}^{G} P_{G, s c h e d, i} \cdot c_{G, i}$ and cost of reserves $\sum_{i=1}^{G} r_{i} \cdot c_{r e s, i}$ are omitted as well as constraints (4) - (7). 


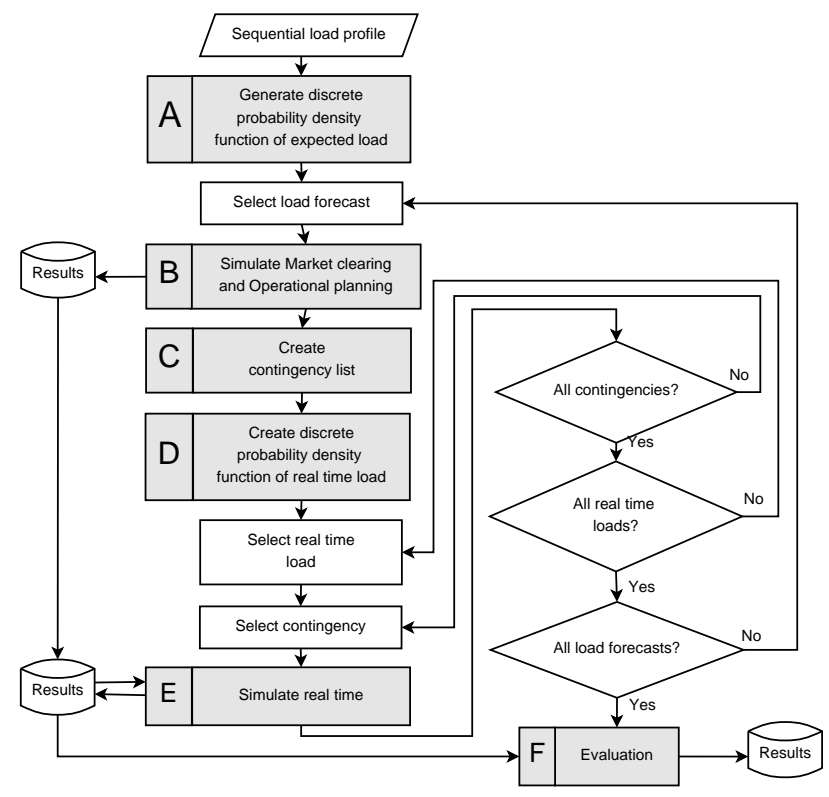

Fig. 4. Flowchart of the evaluation procedure applied in the case study. The letters indicated in the flowchart are referred to in the text.

\section{Evaluation procedure in the case study}

Performance indicators are evaluated on a one year test case using an approximate analytical state enumeration approach, of which the flowchart is shown in Fig. 4. The focus is on system level evaluation rather than on stakeholder level. Forecast of total system load is considered to vary according to a sequential load profile as in Fig. 3(a). A discrete probability density function of the load forecast is made out of this sequential load profile (A) as shown in Fig. 3(c). Operational planning decisions are based on this load forecast (B). Real time load will probably deviate from these forecasts. This can be taken into account by adding a probability distribution of the forecast error $\sigma_{P_{L}}$ on top of the forecast value (D) as shown in Fig. 3(d). A similar approach can be used to include renewable energy sources. Furthermore, the real time operation decision making process for the most probable contingency states up to a particular cumulative probability of occurrence is evaluated (E). These contingency states are selected using a fast sorting technique [30] (C). For the considered system states, reliability actions determined in the quantitative simulation (B and E) are evaluated together with consequences of taking these actions. Outcomes of all considered system states are combined in expected indicator values taking into account probability of occurrence of the load forecast, the real time load given a particular forecast and the contingency $(\mathrm{F})$.

The calculated economic indicator is expected total system cost (ETC):

$$
\begin{aligned}
E T C= & \sum_{s} \operatorname{Pr}_{s} \cdot\left[C_{\text {sched }, s}^{\text {eval }}+C_{r e s, s}^{\text {eval }}+\ldots\right. \\
& \sum_{k}\left[\operatorname{Pr}_{k \mid s} \cdot\left(C_{\text {redisp }, k \mid s}^{\text {eval }}+C_{L C, k \mid s}^{e v a l}\right)\right]
\end{aligned}
$$

with

Cost of scheduled generation: $C_{s c h e d, s}^{\text {eval }}=\sum_{i=1}^{G} P_{G, \text { sched }, i, s} \cdot c_{G, i}$

Cost of reserves: $C_{r e s, s}^{e v a l}=\sum_{i=1}^{G} c_{r e s, i} \cdot r_{i, s}$

Redispatch cost: $C_{\text {redisp }, k \mid s}^{\text {eval }}=\sum_{i=1}^{G}\left[c_{G, \text { red, },}^{+} \cdot \Delta P_{G, i, k \mid s}^{+}+\ldots\right.$

$\left.c_{G, r e d, i}^{-} \cdot \Delta P_{G, i, k \mid s}^{-}\right]$
Cost of load curtailment: $C_{L C, k \mid s}^{e v a l}=\sum_{j=1}^{L} V o L L_{j} \cdot P_{s h e d, j, k \mid s}$

The reliability level of the system is evaluated in terms of expected load curtailment $(E L C)$ :

$$
E L C=\sum_{s}\left[\operatorname{Pr}_{s} \sum_{k} \sum_{j} \operatorname{Pr}_{k \mid s} \cdot P_{\text {shed }, j, k \mid s}\right]
$$

This process is repeated for all reliability criteria on the list, as well as a known criterion, using identical load, generation and grid data.

\section{Illustration of the framework}

The three main parts of the framework, namely simulation of the short term TSO decision making process, performance evaluation and comparison, are illustrated using the basic configuration of the framework explained in section VI.

Appropriate decisions according to the applied reliability criterion can be determined for every possible contingency and real time realization of load. This results in a final system state with a particular reliability level that is obtained at a particular cost. Table III shows average values of actions taken over a whole year in the operational planning stage and real time operation stage. It is clear that decisions taken in operational planning as well as in real time operation depend on the applied reliability criterion, as indicated by the bold elements.

A large share of contingency states has a very low probability of occurrence, but might have large consequences. The probabilistic reliability criterion takes into account uncertainties in a more convenient way by considering various contingencies and different scenarios for real time load and generation with the respective probability of occurrence and severity of the state. The deterministic criteria only look at a prescribed list of credible contingencies at expected load and generation, considering them all equally probable and equally severe. Fig. 5 shows that different reliability criteria lead to different load curtailment risk levels for various real time system states. Fig. 5(a) gives the amount of load curtailed for various real time power system states as a function of probability of occurrence of each state. The evaluation is made for the three reliability criteria using identical contingency states. Lines of constant risk are given for three risk levels, which are useful for assessing the risk level of the power system in various contingency states for various reliability criteria. According 
TABLE III

AVERAGE VALUES OF DECISIONS TAKEN ACCORDING TO DIFFERENT RELIABILITY CRITERIA IN OPERATIONAL PLANNING AND REAL TIME OPERATION. THE BOLD ELEMENTS INDICATE THAT DECISIONS TAKEN DEPEND ON THE APPLIED CRITERION.

\begin{tabular}{|c|c|c|c|c|c|c|c|c|c|c|c|c|c|}
\hline \multirow{2}{*}{$\begin{array}{l}\text { Gen. } \\
\text { no. }\end{array}$} & \multicolumn{3}{|c|}{ Reserves [MW] } & \multicolumn{3}{|c|}{ Schedule [MW] } & \multicolumn{3}{|c|}{ Redispatch [MW] } & \multirow{2}{*}{$\begin{array}{l}\text { Node. } \\
\text { no. }\end{array}$} & \multicolumn{3}{|c|}{ Load curtailment [MW] } \\
\hline & $\mathrm{N}-0$ & $\mathrm{~N}-1$ & Prob. & $\mathrm{N}-0$ & $\mathrm{~N}-1$ & Prob. & $\mathrm{N}-0$ & $\mathrm{~N}-1$ & Prob. & & $\mathrm{N}-0$ & $\mathrm{~N}-1$ & Prob. \\
\hline 1 & 7.80 & 7.80 & 7.73 & 1.37 & 0.64 & 0.39 & 0.01 & 0.01 & 0.01 & 1 & 0 & 0 & 0 \\
\hline 2 & 9.53 & 9.53 & 9.51 & 9.16 & 9.16 & 9.09 & 0.03 & 0.11 & 0.10 & 2 & 0.01 & 0.01 & 0.01 \\
\hline 3 & 0 & 0 & 0 & 9.99 & 9.99 & 9.99 & -0.07 & -0.05 & -0.05 & 3 & 0.059 & 0.053 & 0.054 \\
\hline 4 & 1.53 & 1.53 & 1.53 & 18.45 & 18.45 & 18.45 & -0.02 & -0.007 & -0.006 & 4 & 0.019 & 0.019 & 0.021 \\
\hline 5 & 3.22 & 3.45 & 3.45 & $\mathbf{0}$ & 0.3 & 0.58 & 0.007 & 0.01 & 0.005 & 5 & 0.026 & 0.026 & 0.024 \\
\hline 6 & 9.99 & 9.55 & 9.62 & 5.73 & 6.16 & 6.10 & 1.52 & 1.42 & 1.51 & & & & \\
\hline 7 & 0 & 0 & 0 & 19.98 & 19.98 & 19.98 & -1.52 & -1.53 & -1.52 & & & & \\
\hline 8 & 0.46 & 0.46 & 0.46 & 19.51 & 19.51 & 19.51 & -0.03 & -0.03 & -0.03 & & & & \\
\hline 9 & 2.87 & 2.87 & 2.87 & 17.1 & 17.1 & 17.1 & -0.02 & -0.02 & -0.02 & & & & \\
\hline 10 & 0 & 0.20 & 0.23 & 0 & 0 & 0 & 0 & 0.001 & 0.002 & & & & \\
\hline 11 & 4.56 & 4.56 & 4.56 & 0 & 0 & 0 & 0.013 & 0.012 & 0.008 & & & & \\
\hline
\end{tabular}

to the probabilistic reliability criterion, the risk taken in the preventive stage should be more adapted to the severity and probability of various real time system states. However, Fig. 5(b) and 5(c) show that this does not imply that probabilistic reliability criteria lead to less load curtailment in all real time system states. In order to make a complete evaluation and decent comparison of the performance of reliability criteria, it is important to consider not only the final system state, but also the reliability decision related trajectory according to a particular reliability criterion. Combining both aspects in the evaluation and comparison is one of the features of the presented framework.

Table IV gives an overview of expected total cost and expected load curtailment in the 5 node and 24 node test systems for three different reliability criteria. Indicator values are calculated using (15) and (16). Values are expressed relatively to the indicator value of the reliability criterion with the highest value for the respective indicator. All spare generation capacity was considered to be available for re-dispatch in real time for these simulations. For the analysed systems and system conditions, probabilistic reliability management outperforms deterministic reliability criteria in terms of ETC and ELC, especially in the IEEE 24 node system, which is bigger and has more operational flexibility. Performance of reliability criteria depends on several system parameters, which asks for an analysis of each system individually [13]. In order to make objective and general conclusions about the performance of power system reliability criteria, an assessment of highly influential parameters to consider in the analysis is needed, as well as an impact assessment on higher moments.

\section{CONCLUSION}

Different reliability criteria lead to different decisions, implying different reliability levels and system costs. Comparing performance of reliability criteria is important to convince stakeholders of applying alternative criteria. A framework has been presented that TSOs can use for evaluating and comparing performance of power system reliability criteria in operational planning and real time operation decision making processes. The integrated, generic and modular design used in the presented framework goes beyond existing literature, which focuses on selected issues, without analysing the full

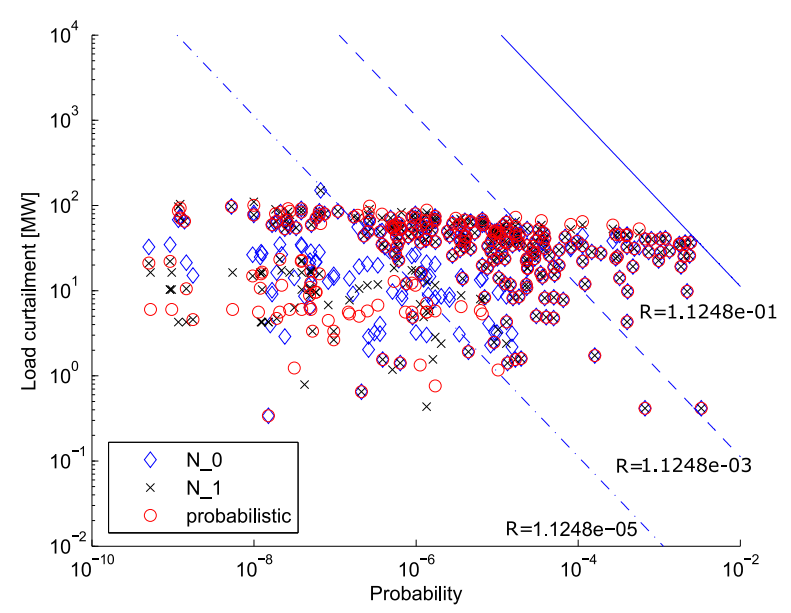

(a) Load curtailment in absolute values. The three lines indicate constant risk with the corresponding risk values indicated at the left of the lines.
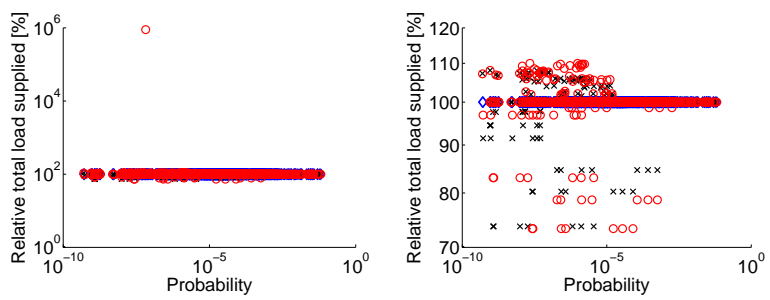

(b) Load supplied according to a par- (c) Zoom of figure 5(b) around 100\% ticular reliability criterion $P_{\text {sup, crit }}$ relatively expressed to the load supplied according to the $\mathrm{N}-0$ criterion $P_{\text {sup }, N-0}$ for different contingency states. In order to avoid division by 0 in cases with no load supplied, following formula is used: $100 \frac{P_{\text {sup }, \text { crit }}+0.01}{P_{\text {sup }, N-0}+0.01}$. Higher values imply less load curtailment.

Fig. 5. Load curtailment in absolute values (Fig. 5(a)) and load supplied, i.e. load demand minus load curtailed, in relative terms (Fig. 5(b) and 5(c)) for various contingency states as a function of probability of occurrence for three different reliability criteria. The legend in Fig. 5(a) holds also in Fig. 5(b) and 5(c). 
TABLE IV

OVERVIEW OF PERFORMANCE OF THREE RELIABILITY CRITERIA FOR TWO TEST SYSTEMS IN TERMS OF COST (TC) AND RELIABILITY (LC). RELATIVE INDICATORS ARE EXPRESSED RELATIVELY TO THE CRITERION WITH THE HIGHEST VALUE OF THE RESPECTIVE INDICATOR.

\begin{tabular}{|c|c|c|c|c|c|c|}
\hline \multirow{2}{*}{$\begin{array}{l}\text { Test system } \\
\text { Criterion }\end{array}$} & \multicolumn{3}{|c|}{ RBTS 5 node } & \multicolumn{3}{|c|}{ IEEE 24 node RTS } \\
\hline & $\mathrm{N}-0$ & $\mathrm{~N}-1$ & Prob. & $\mathrm{N}-0$ & $\mathrm{~N}-1$ & Prob \\
\hline Rel. ETC [\%] & 100 & 99.5 & 98.1 & 100 & 93.8 & 67.1 \\
\hline $\mathrm{P}_{99.5^{\text {th }}} \mathrm{TC}[€]$ & $8.8 \mathrm{E} 4$ & $8.5 \mathrm{E} 4$ & $3.1 \mathrm{E} 4$ & 84.7E4 & 84.7E4 & $43.8 \mathrm{E} 3$ \\
\hline Rel. ELC [\%] & 100 & 98.7 & 97.6 & 100 & 88.0 & 18.4 \\
\hline $\mathrm{P}_{99.5^{t h}} \mathrm{LC}[\mathrm{MW}]$ & 4.76 & 4.76 & 4.26 & 76.8 & 76.8 & 0 \\
\hline
\end{tabular}

reliability problem in an integrated manner. Due to the modular structure of the framework, building blocks can easily be replaced by more elaborated or detailed blocks with the same functionality.

Two test cases show that by using a probabilistic approach, an improvement in both expected cost and reliability can be achieved. The improvement is larger in bigger systems with more operational flexibility. The focus in this paper was on the simulation and evaluation module of the framework, which quantifies performance in terms of reliability and socioeconomic indicators. Further research is needed to develop a good methodology for comparing the overall performance of power system reliability criteria in an objective manner, taking into account influence of different parameters on the performance and the relative importance of these parameters in the result. Furthermore, alternative proxies representing reliability and/or its cost can be determined. An objective of the GARPUR project is to make a practical large scale implementation of a quantification platform, which is based on this theoretical framework. This large scale implementation will apply the methodology in real situations in actual power systems, based on which guidelines towards alternative reliability criteria can be derived.

\section{ACKNOWLEDGMENT}

The authors would like to thank the partners of the Garpur consortium for the fruitful discussions at the project meetings.

\section{REFERENCES}

[1] L. Vanfretti, D. Van Hertem, and J. O. Gjerde, "Smart transmission grids vision for europe: Towards a realistic research agenda," in Smart Grid Applications and Developments. Springer, 2014, pp. 185-220.

[2] D. S. Kirschen, "Power system security," Power Engineering Journal, vol. 16 , no. 5 , pp. 241-248, Oct. 2002.

[3] CIGRE Working Group C4.601, "Review of the current status of tools/techniques for risk based and probabilistic planning in power systems," CIGRE Brochure 434, Oct. 2010.

[4] D. S. Kirschen and D. Jayaweera, "Comparison of risk-based and deterministic security assessments," IET Generation, Transmission \& Distribution, vol. 1, no. 4, pp. 527-533, Jul. 2007.

[5] R. Billinton and R. N. Allan, "Power system reliability in perspective," Electronics and Power, vol. 30, no. 3, pp. 231-236, Mar. 1984.

[6] N. D. Reppen, "Increasing utilization of the transmission grid requires new reliability criteria and comprehensive reliability assessment," in Proc. 2004 Int. Conf. PMAPS, Sep. 2004, pp. 933-938.

[7] J. McCalley, S. Asgarpoor, L. Bertling, R. Billinton, H. Chao, J. Chen, J. Endrenyi, R. Fletcher, A. Ford, C. Grigg et al., "Probabilistic security assessment for power system operations," in Proc. 2004 IEEE Power Engineering Soc. General Meeting, Jun. 2004, pp. 212-220.
[8] J. He, L. Cheng, D. Kirschen, and Y. Sun, "Optimising the balance between security and economy on a probabilistic basis," IET generation, transmission \& distribution, vol. 4, no. 12, pp. 1275-1287, Dec. 2010.

[9] W. Fu and J. D. McCalley, "Risk based optimal power flow," in Proc. 2001 IEEE PowerTech, Sep. 2001.

[10] T. Guler, G. Gross, E. Litvinov, and R. Coutu, "Quantification of market performance as a function of system security," IEEE Trans. Power Syst., vol. 22 , no. 4 , pp. $1602-1611$, Nov. 2007.

[11] G. Kjølle and G. Oddbjorn, "SAMREL: Security of electricity supply-analysis tool," [Online]. Available: http://www.sintef.no/globalassets/upload/energi/nyhetsbrev/ security-of-electricity-supply---analysis-tools.pdf.

[12] Garpur consortium, "ID 3.2.1: Quantification method in the absence of market response," 2015 (Internal deliverable).

[13] E. Heylen, G. Deconinck, and D. Van Hertem, "Impact of value of lost load on performance of power system reliability criteria and their management," in Proc. 2015 IEEE PowerTech, Jun. 2015.

[14] Garpur consortium, "Functional analysis of reliability management," [Online]: http://www.garpur-project.eu/deliverables, 2014.

[15] C. Singh and R. Billinton, "Reliability modelling in systems with nonexponential down time distributions," pp. 790-800, 1973.

[16] J. Endrenyi, Reliability Modeling in Electric Power Systems, ser. A Wiley-Interscience Publication. Wiley, 1979.

[17] M. Khan and R. Billinton, "A hybrid model for quantifying different operating states of composite power systems," IEEE Trans. Power Syst., vol. 7, no. 1, pp. 187-193, Feb. 1992.

[18] G. K. Stefopoulos, F. Yang, G. J. Cokkinides, and A. S. Meliopoulos, "Advanced contingency selection methodology," in Proc. 2005 IEEE 37th Annu. North American Power Symp., Oct. 2005, pp. 67-73.

[19] NERC, "Reliability concepts," [Online] Available: http://www.nerc.com/ files/concepts_v1.0.2.pdf, Dec. 2007.

[20] T. Summers, J. Warrington, M. Morari, and J. Lygeros, "Stochastic optimal power flow based on conditional value at risk and distributional robustness," Int. J. Elect. Power Energy Syst., vol. 72, pp. 116-125, Nov. 2015.

[21] Y. Zhang and G. Giannakis, "Robust optimal power flow with wind integration using conditional value-at-risk," in Proc. 2013 IEEE Int. Conf. on Smart Grid Communications, Oct. 2013, pp. 654-659.

[22] E. Karangelos, P. Panciatici, and L. Wehenkel, "Whither probabilistic security management for real-time operation of power systems?" in Bulk Power System Dynamics and Control-IX Optimization, Security and Control of the Emerging Power Grid (IREP), 2013 IREP Symposium. IEEE, Aug. 2013, pp. 1-17.

[23] A. Monticelli, M. Pereira, and S. Granville, "Security-constrained optimal power flow with post-contingency corrective rescheduling," IEEE Trans. Power Syst., vol. 2, no. 1, pp. 175-180, Feb. 1987.

[24] R. Billinton and L. Wenyuan, "Hybrid approach for reliability evaluation of composite generation and transmission systems using monte-carlo simulation and enumeration technique," in Proc. 1991 IEE Conf. GTD, vol. 138, no. 3. IET, May 1991, pp. 233-241.

[25] R. Billinton, S. Kumar, N. Chowdhury, K. Chu, K. Debnath, L. Goel, E. Khan, P. Kos, G. Nourbakhsh, and J. Oteng-Adjei, "A reliability test system for educational purposes-basic data," IEEE Trans. Power Syst., vol. 4, no. 3, pp. 1238-1244, Aug. 1989.

[26] C. Grigg, P. Wong, P. Albrecht, R. Allan, M. Bhavaraju, R. Billinton, Q. Chen, C. Fong, S. Haddad, and S. Kuruganty, "The ieee reliability test system-1996. a report prepared by the reliability test system task force of the application of probability methods subcommittee," IEEE Trans. Power Syst., vol. 14, no. 3, pp. 1010-1020, Aug. 1999.

[27] M. De Nooij, B. Baarsma, G. Bloemhof, H. Slootweg, and H. Dijk, "Development and application of a cost-benefit framework for energy reliability: Using probabilistic methods in network planning and regulation to enhance social welfare: The n-1 rule," Energy Economics, vol. 32, no. 6, pp. 1277-1282, Nov. 2010.

[28] R. D. Zimmerman, C. E. Murillo-Sánchez, and R. J. Thomas, "Matpower: Steady-state operations, planning, and analysis tools for powe systems research and education," IEEE Trans. Power Syst., vol. 26, no. 1, pp. 12-19, Feb. 2011.

[29] A. Lamadrid, S. Maneevitjit, T. D. Mount, C. Murillo-Sánchez, R. J. Thomas, and R. D. Zimmerman, "A superopf framework," CERTS Report, 2008.

[30] H. Liu, Y. Sun, L. Cheng, P. Wang, and F. Xiao, "Online short-term reliability evaluation using a fast sorting technique," IET generation, transmission \& distribution, vol. 2, no. 1, pp. 139-148, Jan. 2008. 


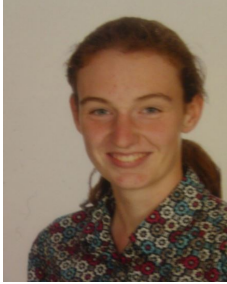

Evelyn Heylen (S'13) was born in 1990 in Belgium. She received the B.Sc. degree in MechanicalElectrical Engineering and the M.Sc. degree in Energy Engineering from the KU Leuven, Belgium, in 2011 and 2013, respectively. Currently, she is working towards a Ph.D. degree at the department of Electrical Engineering of KU Leuven. Her research is funded by a Ph.D. Fellowship of the Research Foundation - Flanders (FWO). Her main area of research interest is power system reliability, focussing on performance evaluation of power system reliability criteria and their management.

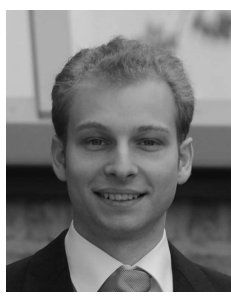

Wouter Labeeuw received the M.Eng. degree in electronics-ict from Provinciale Industriële Hogeschool, Kortrijk, Belgium, in 2007, the M.Sc. degree in computer science from the Katholieke Universiteit Leuven (KU Leuven), Leuven, Belgium, in 2009 and the $\mathrm{Ph} . \mathrm{D}$. degree in engineering, in 2013, also from KU Leuven. He is currently a Postdoctoral Researcher at KU Leuven-ESAT/ELECTA and EnergyVille. His research interests include dat science, demand side management and performance evaluation of power system reliability criteria.

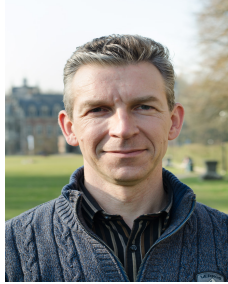

Geert Deconinck (SM'00) is full professor (gewoon hoogleraar) at KU Leuven (Belgium). He received his M.Sc. in Electrical Engineering and his Ph.D. in Engineering Science from the KU Leuven, Belgium in 1991 and 1996 respectively. His research focuses on robust distributed coordination and control, specifically in the context of smart electric distribution networks. Specific interests include smart grids, smart metering, distributed control, robust embedded system design, infrastructure interdependencies and dependability modelling, all in a context of power and communication infrastructures. Both simulation and experimental validation are key. He is head of the research group ELECTA at the Department of Electrical Engineering (ESAT) and is active in the research centre EnergyVille, where he is scientific leader for the research domain 'algorithms, modelling, optimisation', applied to smart electrical and thermal networks. He is chairman of the TI society BIRA on industrial automation and a member of the IEEE SMC TC on Infrastructure Systems and Services, fellow of the Royal Flemish Engineering Society, the Institute of Engineering and Technology (IET), and a senior member of the IEEE (Reliability, Computer and Power \& Energy Societies).

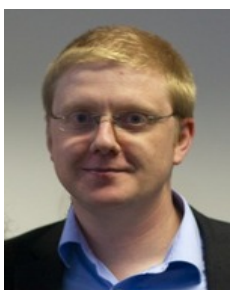

Dirk Van Hertem (S'02-SM'09) was born in 1979, in Neerpelt, Belgium. He received the M.Eng. in 2001 from the Katholieke Hogeschool der Kempen, Geel, Belgium in 2001 and the M.Sc. and Ph.D. degrees in electrical engineering from the KU Leuven, Leuven, Belgium in 2003 and 2009, respectively. His special fields of interest are power system operation and control in systems with flexible ac transmission systems and HVDC and building the transmission system of the future, including offshore grids and the supergrid concept. Since 2011, he has been with the University of Leuven, Leuven, where he is an Assistant Professor in the Electa group. Dr. Van Hertem was a member of the EPS Group at the Royal Institute of Technology, Stockholm, Sweden, in 2010, where he was the Program Manager for controllable power systems for the $E K C^{2}$ Competence Center. He is an active member of the IEEE Power and Energy Society, the Industry Applications Society and Cigré 